\title{
Informe sobre la radio-exposición en el personal quirúrgico de Ortopedia y Traumatología \\ Principios, marco legal y análisis situacional en la Argentina
}

\author{
Mauro Vivas, Horacio Herrera, Juan Pablo Guyot, Gala Santini Araujo, \\ Bibiana Dello Russo, Jorge Castellini \\ Comité de Investigación de la Asociación Argentina de Ortopedia y Traumatología
}

Recibido el 28-1-2018. Aceptado luego de la evaluación el 19-4-2018 • Dr. MAURO VIVAS • vivasmauro@ hotmail.com (1D)

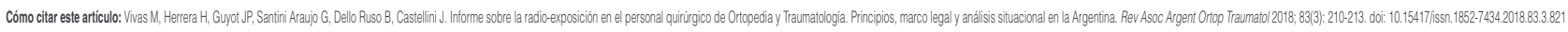

\begin{abstract}
RESUMEN
La cirugía ortopédica ha avanzado mucho en estos años y, con ello, el uso de la intensificación de imágenes en el quirófano. El objetivo de este informe es revisar los principios biológicos de la radiación, el marco legal actual y hacer una breve reseña sobre la situación actual en la Argentina.
\end{abstract}

Palabras clave: Radiación; cirugía ortopédica.

\section{REPORT ON THE RADIATION EXPOSURE IN THE ORTHOPEDIC AND TRAUMATOLOGY SURGICAL STAFF. Principles, Legal framework, and Situational analysis in Argentina}

\begin{abstract}
Orthopedic surgery has significantly advanced in the past years along with image intensifier fluoroscopy in the operating room. The purpose of this report is to review biological principles of radiation, as well as the current legal framework and to outline the present situation in Argentina.
\end{abstract}

Key words: Radiation; orthopedic surgery.

\section{Introducción}

El tratamiento quirúrgico de las fracturas, ya sea mediante técnicas mininvasivas o a cielo abierto, ha logrado mejores resultados funcionales, menor rigidez articular y una mayor satisfacción de los pacientes, si se los compara con los obtenidos clásicamente mediante un tratamiento enyesado con períodos prolongados de inmovilización. Lo propio ocurre en cirugías ortopédicas de reconstrucción en las que, día a día, se logran mejores resultados con procedimientos cada vez menos invasivos.
Estas técnicas han ido ganando aceptación hasta convertirse en el patrón de referencia terapéutico en la actualidad. En consecuencia, la radio-exposición de los pacientes y el personal sanitario aumentó al realizar controles intraoperatorios con aparatos que emiten radiaciones ionizantes. Debemos, por ende, educarnos respecto al correcto nivel de radiación utilizada y la protección para las personas expuestas a la radiación y sus posibles consecuencias (pacientes, personal de quirófano y equipo quirúrgico). El objetivo de este informe es promover el conocimiento sobre las radiaciones ionizantes en el

Conflicto de intereses: Los autores no declaran conflictos de intereses. 
ambiente quirúrgico, en la cirugía de ortopedia y traumatología, las normas nacionales e internacionales que estandarizan el modo correcto de uso, mostrar los resultados del censo llevado a cabo en los Servicios acreditados en la Asociación Argentina de Ortopedia y Traumatología, y el panorama actual de la práctica diaria.

\section{Mecanismo nocivo de la radiación ionizante}

Se denomina radiodiagnóstico al conjunto de procedimientos de exploración y visualización de las estructuras anatómicas del interior del cuerpo humano mediante el uso de rayos $\mathrm{X}$. La continua aparición de nuevas técnicas e indicaciones hace que día a día se incremente el número de actos médicos en los que se utilizan los rayos X. ${ }^{1}$

Las radiaciones ionizantes generadas durante la radioexposición interactúan con los organismos vivos cediendo energía en la masa de estos, produciendo ionización en sus moléculas. Este tipo de energía determina transformaciones físico-químicas que pueden inducir cambios en moléculas biológicamente importantes y estas, por último, efectos biológicos determinantes. La cantidad de energía absorbida por los tejidos expuestos es la que determina el efecto. Se considera que las personas sufren, en promedio, $2,1 \mathrm{mSv}$ por radiación ambiental y, en algunos sectores, pueden sufrir entre 8 y $20 \mathrm{mSv}^{2}$

Los efectos producidos en el órgano expuesto pueden clasificarse en estocásticos o no estocásticos.

Estocásticos: no tienen un "umbral de exposición" y la probabilidad de aparición se considera función lineal de la cantidad de radiación absorbida. Los ejemplos más importantes de estos efectos son la inducción de cáncer y las mutaciones genéticas.

No estocásticos: también llamados agudos; se observan cuando la cantidad de radiación absorbida supera un valor "umbral". Un ejemplo de ellos son las alteraciones en las fórmulas hemáticas.

Los dosímetros para medir la radiación que alcanza al sensor utilizan las siguientes unidades:

Para dosis absorbida, dosis en tejido u órgano: Gray (Gy) (Tabla).

- Equivale a $1 \mathrm{~J} / \mathrm{kg}$

- Unidad anteriormente usada: Rad

- Equivalencia: $1 \mathrm{~Gy}=100 \mathrm{Rad}$

Para dosis equivalente y dosis efectiva: Sievert (Sv)

- Equivale a 1 Gray por factores de calidad de la radiación y tejido.

- Unidad anteriormente usada: Rem

- Equivalencia: $1 \mathrm{~Sv}=100 \mathrm{Rem}$
Tabla. Dosis de radiación absorbida por regiones

\begin{tabular}{|l|c|}
\hline Exploración & $\begin{array}{c}\text { Dosis de la superficie } \\
\text { a la entrada (mGy) }\end{array}$ \\
\hline Abdomen, anteroposterior & 10,0 \\
\hline $\begin{array}{l}\text { Columna lumbar, anteroposterior, } \\
\text { posteroanterior }\end{array}$ & 10,0 \\
\hline Columna lumbar, lateral & 30,0 \\
\hline Columna lumbosacra, lateral & 40,0 \\
\hline Cráneo, anteroposterior & 5,0 \\
\hline Cráneo, lateral & 3,0 \\
\hline Cráneo, posterolateral & 5,0 \\
\hline Mamografía & 10,0 \\
\hline Pelvis, anteroposterior & 10,0 \\
\hline Tórax, lateral & 1,5 \\
\hline Tórax, posteroanterior & 0,3 \\
\hline Dental & 7,0 \\
\hline
\end{tabular}

\section{Entes reguladores}

El Estado argentino, de acuerdo con las recomendaciones de organismos internacionales especializados, ${ }^{3}$ tiene a su cargo informar a la población, habilitar y controlar instalaciones, controlar al personal laboralmente expuesto y al público en general, mediante dos organismos especializados dependientes del Poder Ejecutivo Nacional, uno para las radiaciones originadas por radioisótopos y otro para rayos $\mathrm{X}$.

El primero es la Autoridad Regulatoria Nuclear, creada por Ley Nacional de Actividad Nuclear 24.804 del 2 de abril de 1997 y su Decreto Reglamentario 1390 del 27 de noviembre de 1998.

El segundo es el Ministerio de Salud de la Nación y la función es ejercida según la Ley 17.557/67 y su Decreto reglamentario 6320/68.

\section{Protección radiológica}

La protección radiológica parte de tres principios básicos:

1. Justificación de la práctica: No se justificará ninguna aplicación en la que el beneficio neto obtenible no supere el detrimento que produce. 
2. Respeto por los límites de la dosis: La cantidad de radiación que recibe una persona o un grupo está medida por una magnitud llamada dosis. Existen distintos tipos de dosis según se considere al individuo, a grupos de la población o a la población en general. Por otra parte, se puede considerar al individuo en su totalidad o a ciertos tejidos u órganos. Las autoridades antes mencionadas fijan límites para esas dosis, que deben respetarse, por lo que es preciso contar con sistemas que permitan medir o estimar esos valores.

3. Optimización: La simple observancia de los límites de dosis no es suficiente. Los valores deberán ser reducidos tanto como sea razonablemente posible mediante dispositivos de radioprotección o modificación de las técnicas de trabajo.

Estos principios básicos, desde un punto de vista práctico, pretenden reducir la irradiación externa controlando los siguientes parámetros: ${ }^{1}$

- Distancia: debe ser la máxima posible respecto de la fuente emisora de radiación. Cabe recordar que la dosis disminuye con la distancia (de acuerdo con la ley del inverso del cuadrado de la distancia).

- Tiempo: debe ser el menor posible. La dosis es directamente proporcional al tiempo de exposición, si se reduce este a la mitad. La dosis disminuye de forma proporcional.

- Blindaje: cuando la combinación de tiempo y distancia no reduce la dosis a niveles permisibles, hay que interponer una barrera de material absorbente entre la fuente y el usuario.

Si bien no existen límites de dosis para los pacientes, se han reglamentado niveles de referencia para el radiodiagnóstico y niveles de actividad radiactiva para administrar en los exámenes de Medicina Nuclear. Estos niveles, que se evalúan periódicamente, no se deben superar cuando se realiza una buena práctica.

La recomendación de los organismos internacionales de protección radiosanitaria establecen, en el personal expuesto a la radiación, que la dosis equivalente acumulada en cinco años consecutivos no podrá exceder de $100 \mathrm{mSv}^{4}{ }^{4}$ Esta dosis debe ser distribuida de la manera más homogénea posible, durante dicho período, sin superar nunca $50 \mathrm{mSv}$, en cualquier año.

Sobre esta base, la autoridad nacional en el tema impone que la dosis equivalente integrada en un año no podrá exceder los $20 \mathrm{mSv}$, salvo que la autoridad fije un límite diferente para una instalación. ${ }^{3}$

Por otro lado, la Comisión Internacional de Protección Radiológica ha establecido las normas para la radiación que incluyen los límites de dosificación. ${ }^{5} \mathrm{El}$ límite máximo de dosis anual admisible es de $20 \mathrm{mSv}$ para el cuerpo, $150 \mathrm{mSv}$ para la tiroides o los ojos y $500 \mathrm{mSv}$ para las manos (Directrices internacionales, ICRP). El límite de dosis para el personal no clasificado (por ejemplo, cirujanos) es sólo el 30\% de estos límites (es decir, $150 \mathrm{mSv}$ para las manos). ${ }^{6}$ En Alemania, para el trabajo profesional, el límite es de $500 \mathrm{mSv}$ para las manos, $150 \mathrm{mSv}$ para los ojos y $300 \mathrm{mSv}$ para la tiroides. ${ }^{7}$

De acuerdo con el Reglamento sobre protección sanitaria contra radiaciones ionizantes (RD 783/2001), las personas que trabajan en las instalaciones con riesgo radiológico se clasifican, según las condiciones en que realizan su trabajo, en:

- Trabajadores expuestos: las personas que, por las circunstancias en que se desarrolla su trabajo, sea de modo habitual u ocasional, están sometidas a un riesgo de exposición a las radiaciones ionizantes susceptible de entrañar dosis superiores a alguno de los límites de dosis para miembros del público.

Se dividen en categorías:

Categoría A: pertenecen a esta categoría quienes puedan recibir una dosis efectiva superior a $6 \mathrm{mSv}$ por año oficial, o una dosis equivalente superior a $3 / 10$ de los límites de dosis equivalente para el cristalino, la piel y las extremidades. Quedan incluidos en esta categoría los profesionales que realicen su actividad directamente (sin blindaje estructural) en procesos de: Radiología Intervencionista, Hemodinamia y Urodinamia.

Categoría B: se consideran incluidos en esta categoría a los estudiantes y las personas en formación académica, mayores de 18 años que, durante sus estudios, se encuentren expuestos a radiaciones ionizantes.

Se considera "miembros del público" a: los trabajadores no expuestos, los trabajadores expuestos, fuera de su horario de trabajo, los usuarios de las instituciones sanitarias mientras no estén siendo atendidos como pacientes con fines diagnósticos o terapéuticos y cualquier otro individuo de la población.

Es importante aclarar que, en ninguna norma o ley de radio-exposición, se hace hincapié en el cirujano ortopédico de la misma forma que se menciona a otras especialidades. A pesar de ello, por definición, debemos considerar al ortopedista como Categoría A.

\section{Situación argentina: Censo de noviembre de 2016}

El Comité de Investigación de la Asociación Argentina de Ortopedia y Traumatología llevó a cabo un relevamiento sobre el uso de dosímetros en las instituciones con servicios acreditados por esta Asociación. Consistió en una encuesta formulada por correo electrónico a dichos Servicios, en la cual se evaluó el uso de dosímetros tanto por parte de los médicos del plantel estable, como de los residentes; el período de medición, los controles realizados en los dosímetros, los valores de medición que superaran el permitido y las patologías asociadas a la radiación. 
De las 62 instituciones, respondieron solo $24 \mathrm{y}$, de estas, apenas ocho declararon utilizar dosímetros tanto en médicos de planta permanente como en residentes. En el 80\% de los casos, eran medidos mensualmente por empresas tercerizadas y sólo se registró un caso donde la exposición superaba el valor máximo permitido; no fue notificado ningún caso de enfermedad asociada a la radiación.

En conclusión, podemos afirmar que la encuesta por correo electrónico realizada a los Servicios sobre el uso y registro de los dosímetros en el plantel médico y los residentes de los Servicios de Ortopedia y Traumatología reconocidos por la Asociación Argentina de Ortopedia y Traumatología tuvo escasa respuesta, lo que podría significar que el interés en el tema existe, pero el conocimiento y la responsabilidad de las instituciones sobre controles de aparatos y su buen funcionamiento es insuficiente.
Consideramos que estos resultados son una clara alarma, que demuestran la necesidad de promover el conocimiento sobre la radioprotección en el ámbito quirúrgico; por este motivo, proponemos incrementar nuestro vínculo con la Sociedad Argentina de Radiología que, junto al Ministerio de Salud de la Nación, trabaja en planes de concientización, control de materiales de radioprotección, equipamiento de las instituciones y más normas para incrementar nuestros cuidados en el acto quirúrgico.

Por último, a la fecha, estamos trabajando junto con miembros reconocidos de la Asociación Argentina de Radiología en la redacción del manual de "Buenas prácticas radiológicas". Es nuestra intención que esta publicación sirva de guía y pueda ser utilizada por todos los ortopedistas expuestos a radiaciones ionizantes en el quirófano o en cualquier otro ámbito.

\section{Bibliografía}

1. España: Consejo de Seguridad Nuclear; 2015. Disponible en: https://www.csn.es/descargar-monografía.

2. Dewey P, George S, Gray A. Ionising radiation and orthopaedics. Curr Orthop 2005;19(1):1-12.

3. Valentin J. Las recomendaciones de la ICRP en el presente y el corto plazo. Seguridad Radiológica 2003;22:69-76.

4. ICRP Publication 60: 1990 Recommendations of the International Commission on Radiological Protection. Ann ICRP 1991;21 (1-3):1-201.

5. ICRP Publication 103: 2007 Recommendations of the International Commission on Radiological Protection. Ann ICRP 2007;37(2-4):1-332.

6. Hafez MA, Smith RM, Matthews SJ, Kalap G, Sherman KP. Radiation exposure to the hands of orthopaedic surgeons: are we underestimating the risk? Arch Orthop Trauma Surg 2005;125(5):330-5.

7. Bekanntmachung der Neufassung der Röntgenverordnung. Bundesgesetzblatt 2003;1:604-635. 